\title{
Involuntary Emotional Expressive Disorder: A Case for a Deeper Neuroethics
}

\author{
Peter J. Whitehouse* and Sara Waller ${ }^{\dagger}$ \\ *Departments of Neurology, Psychology, Psychiatry, Neuroscience, Nursing, Organizational Behavior, and Biomedical Ethics, \\ Case Western Reserve University, and Departments of Philosophy and Cognitive Science, Case Western Reserve University,
} Cleveland, Ohio

\begin{abstract}
Summary: Understanding why we produce labels for neuropsychiatric conditions, such as Alzheimer's disease (AD), and how we use those words to tell stories about our brain, as well as which groups control such diagnostic discourse, is important to a wise understanding of our cognitive abilities, their limitations, and even our very human nature. Here, we explore the history and current focus of a newly emerging field called neuroethics and explore its relationship (or lack thereof) to a newly created clinical syndrome called involuntary emotional expressive disorder (IEED). The main argument concerns the lack of neuroethical discussion of issues pertinent to social influences on disease and the construction of professional specialization. We are critical of the processes associated with the creation of both the field and the syndrome, and express concern about their eventual outcomes. The interac-
\end{abstract}

tion of social, political, and business institutions, the inherent interests of the advancement of larger research projects (and the individuals that compose them), their potential for profit, and other incentives to enhance marketability and public attention toward certain research programs will be examined as we discuss the development of the field of neuroethics. Similarly, we argue that these social factors and forces are instrumental in the development of IEED as a recognizable category and condition. Our critique is guided by the hope that through such analyses we can improve our understanding of how we go about our academic activities in cognitive neuroscience and also improve our efforts to help people suffering from neuropsychiatric conditions, such as dementia. Key Words: Neuroethics, IEED, diagnosis, neuropsychiatric conditions, bioethics, dementia.

\section{INTRODUCTION}

Health care professionals often think of understanding genes, neurons, and brain systems as key to comprehending human cognition and improving the quality of life of patients with Alzheimer's disease (AD) and related conditions through facilitating the production of new medications and other biological interventions. Equally important to improving the lives of those who suffer from these conditionsperhaps more important-is understanding why we produce labels for neuropsychiatric conditions, how we use those words to tell stories about our brain, and which groups control such diagnostic discourse.

Here, we explore the history and current focus of a newly emerging field called neuroethics and explore its relationship (or lack thereof) to a newly created clinical syndrome called involuntary emotional expressive disorder (IEED). Neuroethics is a growing subfield of bioeth-

Address correspondence and reprint requests to: Peter J. Whitehouse, MD, PhD, Room 357C, Fairhill Center 12200 Fairhill Road, Cleveland, OH 44120. E-mail: peter.whitehouse@case.edu. ics, which itself is just over 30 years old. Like its parent, neuroethics purports to explore the value implications of developing new technologies in neuroscience: for example, the power of neuroimaging to lend to the understanding and potential control of our minds.

IEED is term a newly created (by a drug company with expert consultants) for a collection of symptoms involving loss of emotional control that is claimed to occur in a variety of brain conditions, including AD. For both the field (bioethics) and the condition (IEED), we will describe how the labeling terms evolved and the current state of their use. With the ultimate aim of improving the quality of life of patients and the contributions to society by the professions involved, we will be critical of the nature of the processes associated with the creation of both the field and the syndrome and express concern about the eventual outcomes of the failure to recognize and resist these processes and the system that bears them.

Our main argument points to the lack of neuroethical discussion of seemingly crucial issues pertinent to the social influences on the construction of diseases and 
professional specializations. We suggest that social forces are artificially and detrimentally narrowing neuroethical discourse.

\section{THE SOCIAL CONSTRUCTION OF AD: MISSING MORAL QUANDARIES}

We believe that there is a need for superordinate conceptual categories that refer to conditions that affect human thinking broadly. All diagnostic terms are invented in service of ameliorating human suffering, but all should be periodically reevaluated as medical knowledge, social need, and political forces change over time. Ideally, reliable and valid diagnostic terms help medical professionals select specific therapies for individual patients. Medical and psychiatric terms have both advantages and drawbacks, at least in their particular historical and cultural contexts.

For example, psychiatry has created and eliminated several disease categories, including the now defunct masochistic personality disorder (or self-defeating personality disorder, which ceased to be treated as real with the advent of the DSM IV ${ }^{1,2}$ ) and homosexuality. Such cases complicate notions of what treatment and cure entail and contribute to cultural contemplation of what mental states are socially acceptable, and what is necessary for disorder and disease evaluation. ${ }^{3}$

\section{History, and current construction, of AD}

Medical terms are no less subject to change under cultural pressure. "Alzheimer's disease is a good illustration of the creationist view in nosology" (as Berrios put it ${ }^{4}$ ). The broader category, dementia, is defined as the loss of cognitive abilities in more than one domain (to distinguish it from diseases that cause intellectual impairment in a single domain, such as language impairment in aphasia, and from acute confusional states associated with metabolic problems, such as delirium associated with hypoglycemia).

What we now call AD was first described at a 1906 meeting by Alois Alzheimer, a German psychiatrist and neuropathologist. ${ }^{5,6}$ Although his first case demonstrated both cognitive difficulties and behavioral abnormalities, the emphasis in the 1970s was on these conditions as disorders of memory, attention, language, and other thinking abilities. AD was once restricted to being a presenile dementia (occurring in patients under the age of 65), and was then broadened to senile dementia of the $A D$ type, and finally was expanded further to the current term (i.e., $\mathrm{AD}$ ).

The major finding of the past 100 years of $\mathrm{AD}$ research is the considerable heterogeneity of genetic (multiple mutations causing some forms), pathological (various microscopic features in varying combinations in various brain regions), and clinical manifestations (different rates of progression in different cognitive and behavioral domains). ${ }^{7}$ Expanded application of the term led to higher public visibility (and fear) and to intensification of research efforts, with expectations for effective and profitable treatments. The growing concern about the health needs of Baby Boomers has led to intense focus on brain health in current and future elders and, arguably, not enough attention to brain health in children. Unfortunately, cognitive impairment and even dementia can occur in children from a variety of causes, ranging from malnutrition to traffic- and war-related injuries-not to mention a growing number of infectious and environmental toxic exposures.

Social reconstruction of the term continues in the present, and some have challenged the word 'dementia' as unnecessarily pejorative. ${ }^{8}$ In Japan, for example, a government-led public information campaign has changed the word used to describe people with ageassociated cognitive impairment. A similar effort to reduce the stigma of the word 'dementia' is under way in Quebec, where a movement by patients and patient-advocates is trying to eliminate the word from psychiatric classifications. ${ }^{7,9}$ Medical classifications and nosology change for political as well as biological reasons.

\section{Social impact}

The care of patients with dementia falls to a variety of medical and nonmedical professions, including (among others) neurology, psychiatry, and geriatrics. Through history and across national boundaries, political conflicts that affect professional claims to diagnostic jurisdiction occur about such issues as whether $\mathrm{AD}$ is a mental illness and whether the noncognitive symptoms deserve as much attention as the cognitive ones. ${ }^{10}$

The durability of this, or any, 100-year-old eponym in light of all the biological and clinical variability that has been described in the ensuing century of research is questionable. Moreover, despite political claims to the contrary, what we currently call AD is not clearly separable from normal aging processes, certainly not clinically: not even using sophisticated neuroimaging and neuropsychological assessment, and not even at autopsy, when counts of pathological features overlap. ${ }^{11,12}$ This suggests that labels separating aging and $\mathrm{AD}$ may be misleading and artificial, and that years of research based on such separation have been guided by a false and politically motivated assumption. Where the neuroethicists sit may well affect how these discussions are framed, and the consequent decisions made.

\section{Lack of neuroethical discourse}

Given the facts of the constructive history and continuing questions surrounding the cohesion of $\mathrm{AD}$ as a single disease, and a literature search revealing no neuroethical discourse on the impact of the label and category of $\mathrm{AD}$ on either patients or the use of research 
funds, we first suggest that neuroethical discourse is too narrow regarding AD. Although the literature reflects neuroethical questions regarding the rightness or wrongness of cognitive enhancement, the use of fetal neural tissue, the equality of distribution of resources, the possibility of obliquely encouraging pregnancies with the goal of marketing aborted fetal tissue, and the social stigmas and standards that demarcate disability, there is no corresponding visibility for questions of the meaningfulness or morality of the proliferation and broadening of disease labels.

Naming and the subsequent distribution of resources toward specific areas of research are neglected topics. Few articles, if any, address the removal of disease titles (except perhaps in the case of autism, which became many diseases requiring subspecialists, and so the move could be construed as in the interests of the scientists working on the disorder) or the merging of areas of subspecialization. The ethic of the discourse seems to be one of expansion, perhaps even capitalistic expansion, and imperialism.

\section{INVOLUNTARY EMOTIONAL EXPRESSIVE DISORDER: LACK OF MORAL ORDER}

Our next argument is for the thesis that 'IEED,' like 'AD,' is a label constructed by social forces serving professional and corporate financial interests, as well as ostensibly helping those who might be categorized as suffering from the condition. These social processes raise similar moral concerns and highlight the need for ethical deliberation. We concurrently suggest that these moral concerns are not usually addressed by neuroethicists, due to the influence of the same field- and disease-constructing social forces.

\section{History, and current construction, of IEED}

Long before IEED was conceived, pseudobulbar affect (PBA) was a recognized neurological syndrome of affect dysregulation characterized by uncontrollable crying and/or laughing and produced by an underlying neurological condition. Charles Darwin, in spite of never completing his medical training, was the first to describe pathologic crying and laughing, noting in 1872 that "... certain brain diseases, such as hemiplegia, brain wasting and senile decay, have a special tendency to induce weeping." ${ }^{13} \mathrm{PBA}$ is a phenomenon in which patients with conditions such as multiple sclerosis or amyotrophic lateral sclerosis cry tears or laugh with minimal provocation and less than expected underlying affect.

Through a coordinated series of drug company-supported expert panels, telephone interviews, multimedia self-study programs, and professional clinically themed meetings, an attempt is being made to redefine (i.e., socially reconstruct) PBA. The attempt is to relabel this relatively rare neurological condition as involuntary emotional expressive disorder (IEED), ${ }^{14}$ with aims, we suggest, that are clearly related to expanding the market for drug therapies. As an article in The New York Times ${ }^{15}$ suggested, IEED is defined broadly enough that it might even include drivers with road rage (http://www. ieed.org/pc/living).

PBA is fairly common in conditions such as $\mathrm{MS}^{16}$ and $\mathrm{ALS},{ }^{17}$ but relatively rare in patients with $\mathrm{AD},{ }^{18}$ whereas the less specific state of emotional incontinence or lability is fairly common. ${ }^{19}$ In our opinion, the best management approach is to inform families that the display of emotions is not associated with a strong underlying affect. This is not to neglect the internal state of the patient, but to educate all concerned that the external manifestations do not match the internal subjective states. Nonetheless, several new and older drugs and drug combinations may help in management of these conditions. ${ }^{20}$

There are very few peer-reviewed articles on IEED; rather, most publications are in journals not associated with professional organizations and may well be supported heavily by advertising dollars from the pharmaceutical companies and not necessarily fully peer-reviewed. We do wonder if this is a case of what has been called disease-mongering, and wonder why no professional neuroethicists have raised the same question.

\section{Social and ethical response}

A review of the literature again reveals that few, if any, neuroethicists are exchanging words on IEED, on the reasons for the new label, or the social and political forces involved in its construction and development. What emotions are controlling, or causing us to neglect, the issues surrounding IEED? Because there is clearly a conflict of interest between the involvement of pharmaceutical companies and the defining of new diseases, we would expect such questions to be raised in the philosophical-ethical tradition. Because research interests do set boundaries of debate, we would expect ethicists to attempt to consider matters from outside such research agendas, because questions of right and wrong, justice and injustice, so often center around those people or things that have been excluded or forgotten or left uncategorized.

We suggest that the lack self-reflexive questions (e.g., what emotional or political, social, economic forces control the construction of IEED?) and meta-level questions (e.g., what principles do we use when we develop the ethical parameters of any disease?) is evidence for a currently shallow neuroethics.

Conversely, IEED may fade into history without adequate consideration of the ethics of disease elimination. We cannot predict that any company will be successful at convincing physicians that IEED is a viable or useful concept, nor that drugs to treat IEED will be approved by the FDA. Nonetheless, IEED should not be eliminated as 
a disorder prematurely; it, like other disorders, should have equal opportunity to undergo the kind of scrutiny that a morally imaginative broader field of neuroethics might provide. The lack of ethical discourse on these matters is a double-edged scalpel.

We will continue by describing what we take to be the social and historical factors that have led to the shallowness of neuroethics-the same constructive forces as those that contributed to the appearance of IEED-and describe what a 'deep' neuroethics might look like.

\section{THE ETHICS OF CREATING NEUROETHICS: HISTORY OF THE CONSTRUCTION OF A FIELD}

Neuroethics can be viewed as a subfield of bioethics, which in turn is encompassed by the more general frame of ethics. Ethics itself has a long and varied history in philosophy, which cannot be reviewed in detail or comprehensively here. Broadly, traditional and contemporary ethical systems take an interest in medical and mental health cases in an attempt to navigate and adjudicate the emergent power of humans to control life and death, quality of life, distribution of resources, rights to govern how one's body is treated and medicated, and the limits thereof.

Meta-ethical questions are also considered important by philosophers, who emphasize the need to examine why we adopt some approaches rather than others, why we accept and/or reject ethical principles and standards, how we can come to understand and free ourselves from (or work well within) the social, political, and power relations that bias our views, and ask from what standpoint ethical decisions are made, and whom they might favor.

These questions can be practical questions of embodiment-addressing our motivating emotions, desires, and the ego-gratification of human beings-or more theoretical questions of principle: how should we go about defining 'justice,' 'equality,' and 'right action' in such a way as to be unbiased, or place bias in the right places? We have suggested that neuroethicists have neglected the meta-ethical realm almost entirely, perhaps due to sociological motives, and such neglect is detrimental to all involved. The following history of the field explains our claim.

\section{The beginning: Potter's bioethics}

In the early 1970s, a concern about the power of biology to influence human lives and the lack of attention to the impact of human behavior on ecosystems led Van Rensselaer Potter to coin the term 'bioethics.' Potter's work was both influenced by his academic research as a cancer biologist on the uncontrolled growth of cells and tissues and inspired by the environmental land ethics of
Aldo Leopold. Potter understood bioethics as broad and interdisciplinary, encompassing a wide range of biological and humanities disciplines, recognizing the chaos and randomness of genetic mutation and human thought, and harnessing our power for the betterment of all. The titles of his two pioneering books, Bioethics: Bridge to the Future and Global Bioethics, are evocative of his broad and long-term thinking in this initial formulation of the field of a bioethics. ${ }^{21,22}$

Potter advocated applying one's ethics, and suggested that a bioethicist should live an environmentally and ethically responsible personal life. He exemplified his own doctrine by becoming an activist concerned about the viability of the human species. ${ }^{23,34}$ One might predict that new fields emerging from bioethics would be equally broad-based and applied. The term deep bioethics was coined (by Potter and one of us, P.J.W.) to denote a field that takes the global point of view of many disciplines, is focused on international and environmental issues, and resonates with the spiritual dimensions of our relationship to nature (for a similar usage, consider deep ecology). ${ }^{25}$ Deep bioethics is lived personally, is expansively applied to many subjects and questions, and is anchored in the larger, long-term interests of the species and the planet.

In this deep bioethics, questioning the framing of one's questions is essential, and ego-gratification or professional advancement are not immediately or obviously ethical. Short-term profit-based endeavors that are potentially destructive to the planet are simply wrong, as is divesting oneself from a debate that may have consequences for the earth's inhabitants. Limiting a field or failing to ask probing questions, intentionally or unintentionally, in the service of egoism, professionalism, or economics is in violation of our deep bioethics. ${ }^{25}$

\section{Anti-Potterian construction}

It was a different and narrowing flow of ideas, however, that merged to form the mainstream of bioethics. The founders of the Kennedy Institute of Ethics, which is housed at Georgetown University, used the term in naming their political and academic organizational endeavors in Georgetown-Washington. The founders of this center were concerned about the value implications of medical technology, particularly those approaches that would alter human reproduction, such as in vitro fertilization. Lawyers joined physicians and philosophers and eventually formed an integrated professional organization called the American Society for Bioethics and Humanities. The so-called Georgetown mantra that emerged from this aggregate of founders focused on principles such as autonomy, beneficence, and justice.

Despite feminist critiques ${ }^{26}$ highlighting the importance of relationships rather than individual rights, and narrative rather than abstract principles, the Georgetown 
principles pervaded the discipline, leading to an alienation of those in fields that question principles as dominant and describe their sociological origins; i.e., those that tend toward meta-ethical critique. Ethicists and social scientists were marginalized, leading to a narrowing of the questions asked and issues raised by professional bioethicists and their cousins, professional neuroethicists. ${ }^{27}$ Thus, the shallowing of neuroethics had begun.

\section{Social and ethical consequences}

The concerns of medicine have become the agenda for bioethics (and neuroethics), ${ }^{28}$ as shown by the typical location of bioethics departments in medical schools. We suggest that this subsequent dependency on medical professions has squelched any original inclinations in bioethics toward activism and challenging the goals and values of the medical enterprise. Much attention is still focused on research ethics, with issues of informed consent, experimental design, and international research. These are indeed important topics, and these areas of inquiry helped facilitate progress by ensuring that medical science was not unduly delayed by restrictive rules and regulations. Scientists at times consider institutional review boards as inefficient and impeding, but for the most part science could move forward with an ethical stamp of approval.

Furthermore peripheral questions in organizational ethics get little attention-for example, the environmental impact of hospitals on their community. ${ }^{29}$ The questions raised by bioethicists who are dependent upon medical schools serve at once to legitimize and highlight current medical research, and to ignore and minimize issues not in the interests of medical progress as defined by current medical practitioners. ${ }^{30}$ Omitted are the views of those in outside disciplines, who may well have a more comprehensive and less interested understanding of these matters. And so bioethics runs shallow.

A notable example of the effect of medicine on the agenda of bioethics was the Human Genome Project, which placed considerable funds in the hands of bioethicists to explore the ethical implications of gene technologies. Although it is undoubtedly exciting that we live in the era in which human being beings mapped their own genome, the short-term and even long-term diagnostic and therapeutic consequences of this accomplishment have been slow to emerge. As a result of the medical agenda, bioethicists spend much more time focusing on the ethical implications of genetic discoveries than on, for example, environmental and public health. Only recently, with the 9/11 attacks and Hurricane Katrina, has any emphasis been placed on the moral implications of bioterrorism (social justice) or global warming (environmental ethics).

The wars that result from competition over energy and water resources and the increasing disruptions of ecosys- tems that lead to changes in the distribution of diseases, such as malaria, will undoubtedly have increasing impact on our health care systems. The exposure, particularly of young brains, to toxic chemicals may well contribute to late-life dementias. The dangers of lead, for example, have been well known for centuries, and yet lead abatement is seemingly not on the agenda of neuroethics. Should it be?

\section{Focus on neuroethics}

Neuroethics emerged directly from researchers in the cognitive neurosciences (e.g., M.J. Farah, M.S. Gazzaniga), who rightly came to recognize that the human power to know, and to alter, human brain and behavior has outstripped our guidelines for the ethical applications of our technology. Neuroethicists tend to explore the implications of measuring brain states, leading to questions of privacy and responsibility to inform the public of sinister thoughts, and enhancing brain function, leading to questions of distribution of resources and of prioritization of treatments for mental dysfunction.

Although such questions are important and interesting, we believe that the political origins of this field alter and narrow the questions that neuroethicists ask in a detrimental way. To consider the political soil that germinated neuroethics, we note that one mark of the progress of an academic discipline is often considered to be the creation of subdisciplines: as the field becomes more refined, the questions asked by it become more and more specialized, thus signifying a deepening understanding of the broader category, and productivity of employed research methods. (The parent field, bioethics, is also involved in other manifestations of academic maturity, such as the creation of freestanding departments and Ph.D. programs.) Neuroethics, then, arises from political currents out of a field already constrained by interests and funding desiderata of medical professionals.

Furthermore, neuroethics also emerged as a political counterbalance to the genetic emphasis (or genethics) that has so dominated mainstream bioethics. This neuroethicist view suggests that our brains have as much, or more, to do with determining our human nature and health as do genes. This view both legitimizes bioethics by showing its specialization, and legitimizes neuroethics by emphasizing the difference in issues between the two fields-suggesting that both are indeed necessary research endeavors. However, a critical field such as ethics should be doing more than legitimizing the disciplines that it takes as its focus: this reciprocal legitimization is fundamentally circular and self-perpetuating. Such parasitism does not take a broad, multidisciplinary view of the framing of ethical questions or the ethics of the creation of the new field, and suggests a shallow neuroethics. The place of neuroethics in relation to the 
whole of the relevant intersecting disciplines is quite neglected.

\section{Spotlight on missing issues}

What are the consequences of such political factors in the birth of this new field? Although questions in neuroethics are well centered in the ethical tradition on issues of human power to control mind and behavior, to enhance cognitive abilities, the distribution of such enhancement, rights to govern how one's mind is treated and medicated, privacy of thought, and the limits thereof, we suggest that important questions (of conflict of interest, power relations, the proper home and credentials for the practice of neuroethics, and the power of claiming explanatory value) are missing from the forum of discussion. This list of missing issues is not comprehensive, but it does sketch a domain of serious meta-ethical questions that is Potter-esque in that it is much broader, more interdisciplinary, and concerned with the personal application of principles than we have seen neuroethicists draw to date. In the concluding portion of this article, we examine three important, and relatively untreated issues, using the example of IEED.

\section{IEED AS A MIRROR OF SHALLOW NEUROETHICS}

\section{Control of concepts}

First, one of the major ethical concerns in medicine generally is that health industries such as pharmaceutical and technological companies are dominating medical education (through access and funding) and even our very conceptions of health and disease (through their ability to prescribe medication and develop equipment for detecting for symptom aggregates and thus suggest that these aggregates indicate real diseases). Bioethicists (and possibly, neuroethicists) qua medical professionals potentially receive grants from, and work for, an industry with unclear guidelines for understanding their own roles and own potential conflicts of interest. They have few criteria for accepting and/or questioning the disease paradigms taught in medical training, the accepted courses of research action sponsored by those grants, or the power and wealth of pharmaceutical companies to frame our worldview of mental and physical illness and health.

The emergence of IEED, and the relative silence of neuroethicists on the subject, suggests that neuroethicists have accepted (with few public questions) the dominant paradigm involving the reification of medical disease labels and do not recognize that they participate in the social machinery of the medicalized research world by accepting the frame in which the disease is presented. As neuroethicists currently do ask traditional questions of patient rights and privacy, the enhancing of cognitive powers, and the distribution of resources, they are easily dissuaded from asking questions about IEED, which is seldom so severe in its own right as to impact patient rights (having the condition does not make one incompetent) or to be a disorder that runs at the forefront of questions of 'mental enhancement' (having more emotional control is not as clearly desirable as having an enhanced memory or executive function-indeed, social intelligence depends on emotional awareness). IEED does not clearly raise questions of resource distribution, because the treatments are not yet on the market and their costs are not yet decided. The neuroethical silence regarding the uncritical acceptance of the medical paradigm both fails to take a multidisciplinary view on its own position in the professional world, which suggests the second issue: qualifications.

\section{Qualifications for neuroethicists}

What training is proper to qualify one as a neuroethicist? We can consult lawyers about legal aspects and religious leaders about spiritual matters, but ethical quandaries in specialized fields of neurobiology and medicine point to practitioners with clinical experience as well as philosophers with theoretical expertise. Surely such decisions need to be informed ones, but the information is distributed across a number of fields. What justificational ground does any expert have to lay claim to these decisions and suggest superiority of position or training over any other related expert? What should the credentials for a neuroethicist entail?

Neuroethics again seems silent on this question set, failing to rise to the meta-level and ask questions about itself, perhaps because the current professional neuroethicists have a conflict of interest themselves: that is, they are professionally motivated not to ask such questions, given the social circumstances surrounding the development and maintenance of the field. We suggest that deeper neuroethicists should recognize the self-referentiality of their own field and should therefore ask questions about who they are as embodied humans with neurological powers and constraints, as people with interests and social and economic relationships, and with biases that will inevitably guide them as they make decisions about how moral decisions are understood by our community. Neuroethicists and neuroscientists have an interest in the flourishing of neuroethics, and thus the criteria for becoming a neuroethicist should be well delineated and carefully conscribed, lest our neuroethical questions be stunted or distorted.

In this regard, neuroethicists could themselves be exhibiting a scholarly form of IEED, in that the direction and aims of their discourse and literature involuntarily reveal their hidden professional and emotional commit- 
ments. In fact, neuroethicists have emerged from, and are in danger of being governed by, medical and neurological commitments. The failure to express concern regarding the qualifications and position of those who have developed IEED is a mirror of the lack of concern regarding the qualifications and position of those who have developed neuroethics.

\section{Need to recognize "neuro-" power}

Third, the social power of placing "neuro-" in front of any word to legitimize the endeavor as scientific and respectable has gone unexamined and unquestioned, as has the impetus to co-opt other fields as subordinate to neuroscience (neuroeconomics, neuropolitics, neuropsychotherapy, neurophilosophy, neurotheology, neurolaw). By claiming that there is an ethical issue created by some scientific invention or discovery, one is essentially intensifying its social power. Visibility leads to government and private funding, which leads to further discoveries and an expansion of the field. As the field expands, it appears to have more consequences in ordinary lives, where ethical decisions matter, and across disciplines, invading and explaining humanities and social sciences alike with a reductionistic spirit.

Nonetheless, just as we have found no neuroethical literature on the legitimizing of diseases such as IEED through the development and proliferation of labels for them, we have found no neuroethical literature on the topics of the social and political force of the "neuro-" affix or the subsumption of other fields as explicable by neuroscientific methods. Indeed, practitioners of neuroethics may have a professional interest in neglecting to ask such questions, because the state of the field as it is can only intensify and facilitate their power to make decisions and create moral frameworks.

\section{CONCLUSIONS}

Creating labels for disease conditions and for the disciplines that act in the field of health and human values are key social, political, and economic strategies informed by science and clinical practice-but, ideally, not ultimately controlled by them. Hence inventing the terms IEED and neuroethics represents fundamentally the same human social cognitive process: the social construction of the language we use to organize our communal activities in service of ameliorating human suffering. The question is whether neuroethics can help in this enterprise.

Neuroethics could better serve the medical profession and society by asking deeper questions about the ethics of our methods for understanding brain aging and disease. It could question the application of uncertain labels and the social value of expenditures dealing with genetic and imaging technologies. It could inquire about the important health implications of environmental deterioration. A neuroethics conceived in the Potterian way could have a profound impact on the quality of lives of human beings and other species. On the other hand, if it continues on its current course, neuroethics is likely in the long run to focus our attention in narrow, myopic places, to have us waste money, and, by consequence, to have a net negative consequence of our species and our planet.

We have argued that the priorities of neuroethicists lie with the expansion and further development of their field, and that the ethical issues they raise are too limited. What are the ethics of neuroethics? Many gray matters have been left rather dark.

Acknowledgments: The authors express their gratitude to Peter Rabins for help with the references.

\section{REFERENCES}

1. Caplan P, Gans M. Is there empirical justification for the category of 'self defeating personality disorder'? Fem Psychol 1991;1:263278.

2. American Psychiatric Association. Diagnostic and statistical manual of mental disorders (DSM-IV), 4th ed. Washington, DC: APA; 1994.

3. Waller S. The Wisconsin Card Sort: an empirical and philosophical analysis of presuppositions regarding flexibility of cognition. In: Figueroa R, Harding S, Science and other cultures: issues in philosophies of science and technology. New York: Routledge, 2003: 189-200.

4. Berrios GE. Alzheimer's disease: a conceptual history. Int J Geriatr Psychiatry 1990;5:355-365.

5. Alzheimer A. Über eine eigenartige Erkrankung der Hirnrinde [In German]. Allg Z Psychiatrie 1907;64:146-148.

6. Alzheimer A, Stelzmann RA, Schnitzlein HN, Murtagh FR. An English translation of Alzheimer's 1907 paper, "Uber eine eigenartige Erkankung der Hirnrinde." Clin Anat 1995;8:429-431.

7. Whitehouse PJ, Maurer K, Ballenger J, editors. Concepts of Alzheimer disease: biological, clinical, and cultural perspectives. Baltimore: Johns Hopkins Press, 2000:48-128.

8. Sachdev P. Is it time to retire the term "dementia?" J Neuropsychiatry Clin Neurosci 2000;12:276-279 [Available at http:// www.neuro.psychiatryonline.org/cgi/content/full/12/2/276].

9. Whitehouse PJ. The end of Alzheimer disease. Alzheimer Dis Assoc Disord J 2001;15:59-62.

10. Gaines AD, Whitehouse PJ. Building a mystery: Alzheimer disease, mild cognitive impairment, and beyond. Philos Pychiatry Psychol 2006;13:61-74.

11. Morris JC. Mild cognitive impairment is early-stage Alzheimer disease. Arch Neurol 2006;63:15-16.

12. Whitehouse PJ, Juengst ET. Anti-aging medicine and mild cognitive impairment: practice and policy issues for geriatrics. J Am Geriatr Soc 2005;53:1417-1422.

13. Darwin C. The expression of the emotions in man and animals. New York: Appleton; 1873.

14. Cummings JL, Arciniegas DB, Brooks BR, et al. Defining and diagnosing involuntary emotional expressive disorder. CNS Spectr 2006;11:1-7.

15. Pollack AW. Marketing a disease, and also a drug to treat it. New York Times. May 9, 2005 [Available at http://www.nytimes.com].

16. Panitch HS, Thisted RA, Smith RA, et al. Randomized, controlled trial of dextromethorphan/quinidine for pseudobulbar affect in multiple sclerosis. Ann Neurology 2006;59:780-787.

17. Brooks BR, Thisted RA, Appel SH, et al. Treatment of pseudobulbar affect in ALS with dextromethorphan/quinidine: a randomized trial. Neurology 2004;63:1364-1370. 
18. Starkstein SE, Migliorelli R, Teson A, et al. Prevalence and clinical correlates of pathological affective display in Alzheimer's disease. J Neurol Neurosurg Psychiatry 1995;59:55-60.

19. Arciniegas DB, Lauterbach EC, Anderson KE, et al. The differential diagnosis of pseudobulbar affect (PBA): distinguishing PBA among disorders of mood and affect. Proceedings of a roundtable meeting. CNS Spectr 2005;10:1-14.

20. Avanir Pharmaceuticals. A national discussion about a debilitating condition. PBA Update 2005;2(February issue).

21. Potter VR. Bioethics: bridge to the future. Englewood Cliffs, NJ: Prentice-Hall, 1971.

22. Potter VR. Global bioethics: building on the Leopold legacy. East Lansing, MI: Michigan State University Press, 1988.

23. Whitehouse PJ. The rebirth of bioethics: extending the original formulations of Van Rensselaer Potter. American J Bioethics 2003; 3:W26-W31.
24. Whitehouse P. Van Rensselaer Potter: an intellectual memoir. Camb Q Healthc Ethics 2002;11:331-334.

25. Potter VR, Whitehouse PJ. Deep and global bioethics for a livable third millennium. Scientist 1998;12:1-9.

26. Tong R. Teaching bioethics in the new millennium: holding theories accountable to actual practices and real people. J Med Philos 2002;27:417-432.

27. Murphy TF. Representation and balance on presidential commissions. Am Philos Assoc Newsl Philos Med [online serial] 2005; 04 [Available at: http://www.apa.udel.edu/apa/publications/ newsletters/MedicineNL/v04n2.htm].

28. Illes J, Racine E. Imaging or imagining? A neuroethics challenge informed by genetics. Am J Bioethics 2005;5:5-18.

29. Pierce J, Jameton A. The ethics of environmentally responsible health care. New York: Oxford University Press, 2001.

30. Baker R. A draft model aggregated code of ethics for bioethicists. Am J Bioeth 2005;5:33-41. 